\title{
The Design of Anti-aliasing Analog Filter for Data Acquisition in the Surface Measurement
}

\author{
Xu Jingbo ${ }^{1,2} \mathrm{Xu}$ Xiaohong $^{3}$ Yang Pengyu $^{4}$ Lin Haijun $^{1,2}$ and Guo Xin ${ }^{1,2}$ \\ 1.College of Measure-control Technology \& Communication Engineering, Harbin \\ University of Science and Technology, Harbin, China; \\ 2. The higher educational key laboratory for Measuring \& Control Technology and \\ Instrumentations of Heilongjiang Province, Harbin, China; \\ 3. Department of Textile Engineering, Shazhou Professional Institute of Technology, \\ Zhangjiagang, China \\ 4.Harbin Railway Company, Harbin Railway Administration, Harbin China \\ hitxjb@126.com
}

\begin{abstract}
In the surface measurement system, the data acquisition is key part and the anti-aliasing analog filter is necessary. This paper deals with the design of the anti-aliasing analog filter. Based on the principle of anti-aliasing filtering, the parameters of filter are computed, the filter circuit is designed, and the frequency characteristic is drawn. Combined with digital filter, the filtering can maintain the low frequency components very well and suppress effectively the high frequency signals in the original surface profile, which reduces the distortion caused by noise and makes the filtering effect better. This method has been applied in the surface measurement system and the actually measured data verified the performance of the filter.
\end{abstract}

Keywords: surface measurement, analog filter, filter design, anti-aliasing

\section{Introduction}

The surface measurement is an important issue of geometric measurement field and the surface quality can be accurately assessed through surface measurement. Based on the sensing mechanism, surface measurement methods mainly include non-contacted measurement and contacted measurement. Non-contacted measurement mainly includes object optical grating method, sine wave phase modulation method, light bulb phase-shift interfering method and so on, which has advantages of non-contacted, high sensitivity and high precision, but the price is expensive and the requirements for measurement environmental are very high. Contacted measurement refers to a stylus contacting directly with the measured surface, which is easy to damage the surface and the measuring precision has certain restriction affected by the sensor resolution ${ }^{[1-3]}$. However, for the contacted measurement, the principle is relatively simple, the measurement process is rapid and convenient, the measurement precision can satisfy general requirements, operation is easy and the using cost is low, so it is more common in use.

Whether the non-contacted measurement or the contacted measurement, the data acquisition is a key part of surface measuring instrument. In order to obtain the effective surface profile, the data acquisition system must have enough high sampling precision and 
rate. The electrical system contains high frequency noise, in order to prevent the introduction of noise; it needs to design an anti-aliasing filter to suppress interference.

\section{Principle of Anti-aliasing Filtering}

In the data acquisition system, because the spectrum of the sampled signal repeats on a cycle of $f_{s}$ and the spectrums of adjacent cycle are symmetric around $\frac{2 k-1}{2} f_{s}(k=1 \cdots n)$, if the sampling frequency is not enough high, it will resultin the aliasing phenomenon in spectrum. So $f_{s}$ should conform to the Nyquist theorem, namely to $f_{s} \geq 2 f_{h}$, in which $f_{h}$ means the highest frequency of sampled signal. In the sampling process, the high frequency interference signals are inevitable and also symmetric around $f_{s} / 2$, it could be folded to the low frequency band and form the false frequency components ${ }^{[4-5]}$. Therefore, in the design of the data acquisition system, in order to suppress the occurrence of this phenomenon, it needs to adopt the anti-aliasing filter.

The traditional method is only using low pass anti-aliasing analog filter and the design requirements for the analog filter are relatively high: the pass band ripple should be enough low, the stop band attenuation should be enough high and the transition band should have good steepness ${ }^{[6]}$. Furthermore, the parameters of the analog filter are not easy to adjust, the cost of hardware is high, and it is difficult to adapt to the change of object.

This paper adopts the combination scheme of analog filter and digital filter. This method reduces the requirements of analog filter and makes it easier to implement. The digital filter can make up for the analog filter and improve the overall performance of the filter. Figure 1 shows the diagram. The digital filter is easy to design through FDAtool of Matlab. This paper focuses on the analog filter.

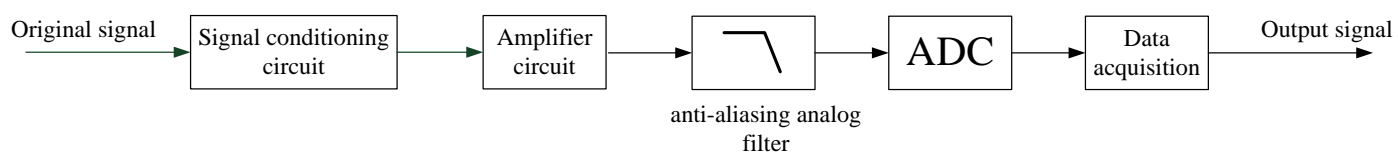

Figure 1. The Block Diagram

The amplitude-frequency characteristic of the traditional anti-aliasing analog filter is shown in Figure 2(a). It can be seen that not only the ripple characteristics in pass band but also the attenuation characteristics in stop band are required. And the transition band should have some gradient, which usually leads that the attenuation in stop band is not enough and the ripple in stop band is larger. So the design requirements of traditional anti-aliasing analog filter are higher, and it is difficult to achieve.

In the new method, in order to lower the design requirements of analog filter, on the condition that Nyquist theorem, through appropriately increasing the sampling frequency and broading the transition zone, the order of filter which can achieve the equal stop band attenuation will reduce and the stop band ripple will decrease, that is shown in Figure 2(b). As can be seen from the figure, the new method requires $f_{s} \gg>2 f_{h}$. In the pass band $0<f<f_{h}$, the filter should have the flat 
amplitude-frequency characteristic; the fluctuation to the input signal should not exceed 1LSB of AD converter. In the stop band $f>f_{s}-f_{h}$, it requires that the filter has a sufficient attenuation, that is, the amplitude attenuation of signal should reach 1LSB of $\mathrm{AD}$ converter. Although the band $f_{s}-f_{h}<f<f_{s}$ maps to $0<f<f_{h}$ in data acquisition system due to aliasing mapped to map to, but because of the effective attenuation of analog filter in this frequency band, the high frequency interference signal does not affect the measured signal. In the transition band $f_{h}<f<f_{s}-f_{h}$, it does not require the filter has certain gradient. In this spectrum band, although the interference signal could not be effectively suppressed and the frequency band $f_{s} / 2<f<f_{s}-f_{h}$ aliases to $f_{h}<f<f_{s} / 2$, but we can restrain the signal in $f>f_{h}$ by designing a high performance low pass digital filter which has the pass band $0<f<f_{h}$. In the new method, the transition band of analog filter is wider and it's steepness is smaller, thereby guaranteeing the attenuation characteristics in stop band, thus the design requirements of the analog filter is reduced, which makes it easy to realize.

In this new method, analog filter is essential. If the analog filter is not used, the high frequency signal in $f_{s}>f>f_{s}-f_{h}$ will alias to the frequency band $0<f<f_{h}$ through digital sampling which can't be filtered effectively only by digital filter. If only dependent on analog filter, the design requirements for the analog filter are higher, and its design and implementation are more difficult. This method, by combining the analog filter and the digital filter, reduces the requirements of analog filter and makes the analog filter easier to implement, and also improves the overall filtering performance.

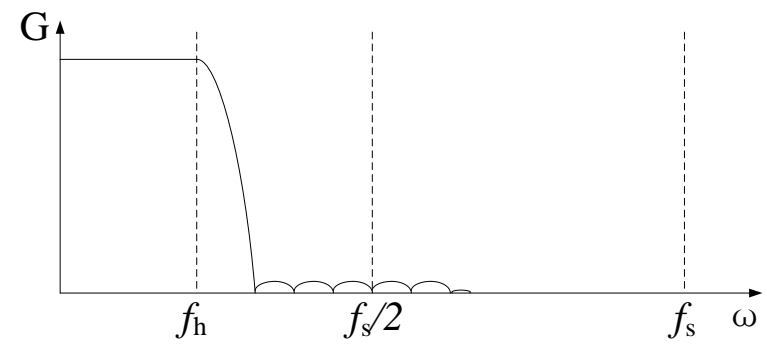

a) The amplitude-frequency characteristic of anti-aliasing analog filter in the conventional method

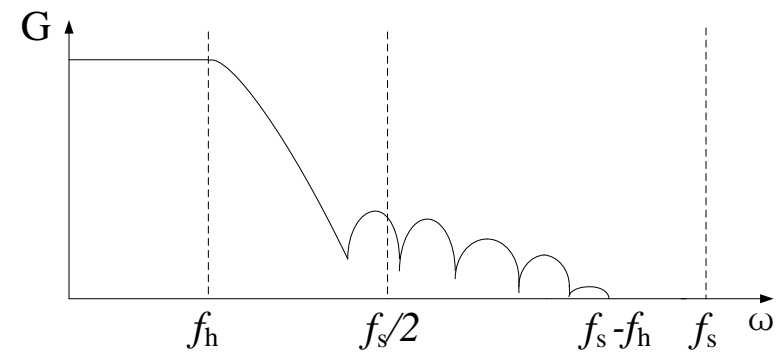

b) The amplitude-frequency characteristic of analog filter in the new method

Figure 2. The Comparison of the Analog Filters in the New Method and the Conventional Method 
Based on that the signal should not be attenuated through the pass band of the filter, setting the maximum amplitude of measured signal is $V_{s}$, namely the full-scale input range of sensor, and the maximum quantization level of $\mathrm{AD}$ converter is $\mathrm{E}$, then that is:

$$
V_{s}=E
$$

Based on that the stop band attenuation of filter should reach to some degree, setting the maximum amplitude of high frequency noise is $V_{n}$, through the stop band attenuation, it should reach the minimum quantization level $q$. Setting the stop band attenuation coefficient is $K_{n}$, that is

$$
V_{n} \cdot K_{n}=q
$$
is:

Coupling the equation (1) and (2), and performing logarithmic operation, the result

$$
-20 \lg K_{n}=20 \lg \frac{E}{q}-20 \lg \frac{V_{s}}{V_{n}}
$$

Because of $E=2^{m} q$, in which $\mathrm{m}$ is the digits of AD converter, then:

$$
-20 \lg K_{n}=6 m-20 \lg \frac{V_{s}}{V_{n}}
$$

\section{The Design of Anti-aliasing Analog Filter in Surface Measurement System}

In fact, in the surface measurement system, the signals above $400 \mathrm{~Hz}$ are false signals caused by electrical components and are not the signals produced by the measured surface, which must be removed from the measured signal ${ }^{[7]}$. The above mentioned anti-aliasing filter has been adopted in the surface measurement system to restrain the noise signal and retain the effective signal below $400 \mathrm{~Hz}$.

Firstly the low pass analog filter needs to be designed. Its performance usually includes the cutoff frequency or the upper and lower boundary frequency, pass band ripple and stop band attenuation; And the approximation type of filter usually consists of Butterworth, Chebyshev I, Chebyshev II, Elliptic and Bessel filter ${ }^{[8]}$.

1) Butterworth low pass filter: that has the flat frequency response in pass band, the monotonous attenuation of stop band is much steeper than Bessel filter, but the phase response is nonlinear with frequency.

2) Chebyshev low pass filter: that has equivalent constant amplitude ripple and better selectivity, but the phase and group delay characteristics are not very good. If the requirements for attenuation and phase characteristics are not very strict, the Chebyshev filter can be chosen.

3) Bessel low pass filter: that has linear phase response, no ripple in pass band and monotonic decay in stop band, but its cut-off characteristic is rather poor, suitable for the signal such as square wave and triangle wave which have wide spectrum.

4) Elliptic low pass filter: that has nearly flat frequency response in pass band, and the attenuation in stop band is relatively steep. In the condition of same order, it has minimum ripple in pass band and stop band. The cutoff characteristic is better than other approximation filters. 
Comprehensive consideration of the above mentioned, the elliptic filter is best choice for designing the anti-aliasing filter, which has favorable amplitude-frequency characteristic and phase-frequency characteristic in pass band from the point of approximation.

The amplitude-frequency characteristic of elliptic filter is:

$$
|H(j \omega)|^{2}=\frac{1}{1+\varepsilon^{2} R_{n}^{2}(\omega)}
$$

In the formula, $\varepsilon$ is the ripple coefficient and $R_{n}(\omega)$ is rational function.

$$
R_{n}(\omega)=\frac{\omega\left(\omega_{1}^{2}-\omega^{2}\right)\left(\omega_{2}^{2}-\omega^{2}\right) \cdots\left(\omega_{k}^{2}-\omega^{2}\right)}{\left(1-\omega_{1}^{2} \omega^{2}\right)\left(1-\omega_{2}^{2} \omega^{2}\right) \cdots\left(1-\omega_{k}^{2} \omega^{2}\right)}
$$

If $n$ is odd, $k=(n-1) / 2$ and if $n$ is even, $k=n / 2$.

Here, an actual measurement process to measure the surface of a work piece is discussed. The cutoff wavelength $\lambda_{c}$ is $0.8 \mathrm{~mm}$, the sampling interval is $0.5 \mu \mathrm{m}$, and there are 1600 sampling points in cutoff wavelength. In $7 \lambda_{c}$, there are 11200 measured data in total. The travelling speed of stylus is $0.85 \mathrm{~mm} / \mathrm{s}$, so the sampling frequency is $1700 \mathrm{~Hz}$. The range of stylus displacement sensor is $10 \mu \mathrm{m}$ and its vertical resolution is $0.0025 \mu \mathrm{m}$. By 12 bits AD converter sampling, the primary profile is shown in Figure 3.

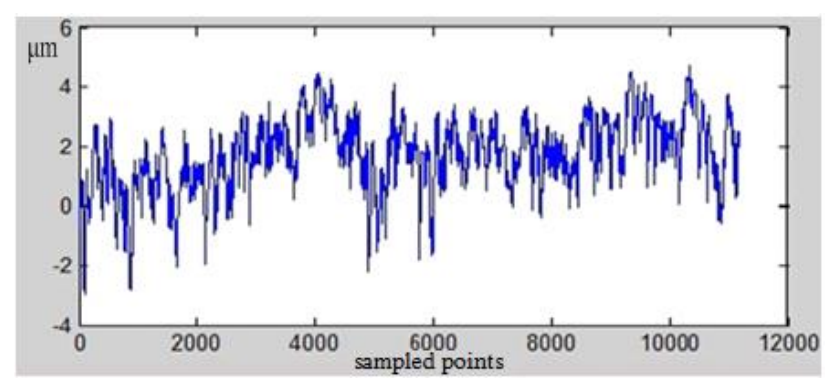

Figure 3. The Primary Profile of Work Piece Surface

If the pass band frequency is $400 \mathrm{~Hz}$, then the stop band frequency is $1300 \mathrm{~Hz}$. In the data acquisition system, on the condition that a 12 bits $\mathrm{AD}$ converter is used, the maximum range of sensor is $10 \mu \mathrm{m}$, and the resolution is $0.0025 \mu \mathrm{m}$, setting the maximum amplitude of noise is 40 times the resolution, through the formula (4), the obtained minimum stop band attenuation should be $-30 \mathrm{~dB}$.

The 6 order elliptic filter is designed and the parameters of $\mathrm{RC}$ are computed by the aided design software Filter Wiz Pro, which is implemented by three cascaded active filters, as shown in figure (4).In the software, the parameters of filter are inputted, then the corresponding design results are drawn. The circuit (a) and (b) are second order low pass filters with the structure of Sallen-Key I. The circuit (c) is second order low pass filter with the structure of Sallen-Key II. The Sallen-Key filter has precision gain and needs less electronic components.

If setting that the stop band frequency of anti-aliasing filter is $430 \mathrm{~Hz}$, that means more narrow transition zone, then the order of filter will attain 12.So that the new method widens the transition zone, reduces the order, and makes the analog filter easy to implement. 


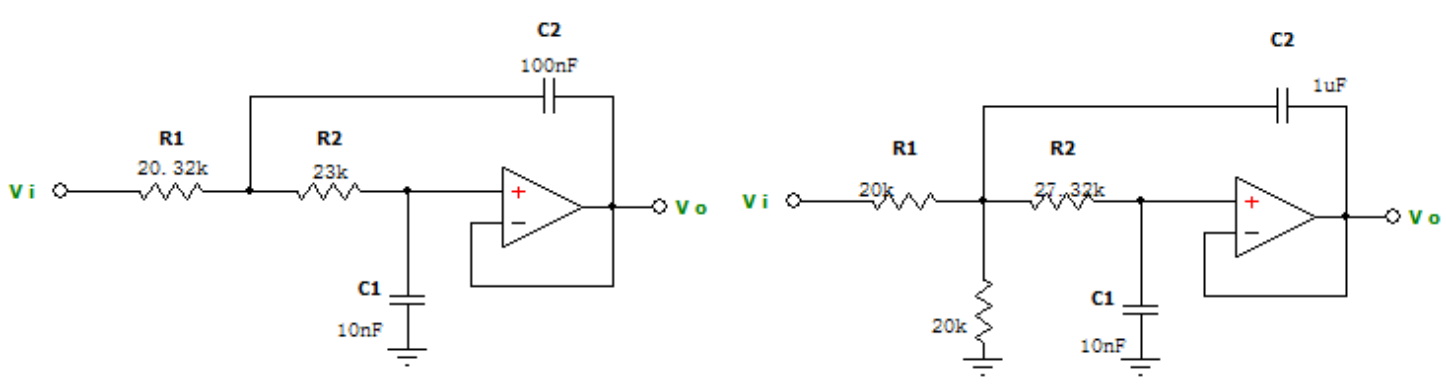

a) The first stage circuit

b) The second stage circuit

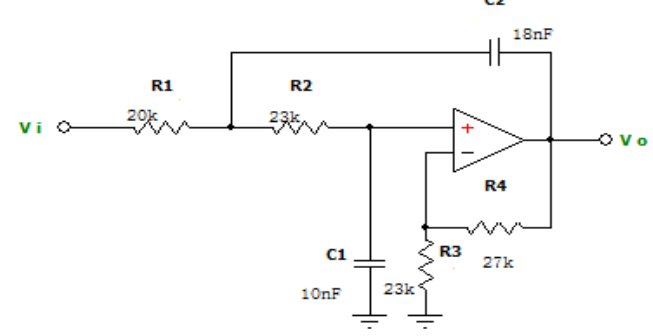

c) The third stage circuit

Figure 4. Three Stages Circuit of Analog Filter

The amplitude-frequency response and the phase-frequency response are shown respectively in Figure (5) and Figure (6). In fact, the analog filter implemented by circuit can only approximate the ideal characteristic and it has usually certain attenuation in the pass band. In Figure (5), the red curve is the ideal amplitude-frequency response and the black curve is the actual amplitude-frequency response. It can be seen that the designed analog filter meets the requirements, has little distortion and its pass band is relatively flat.

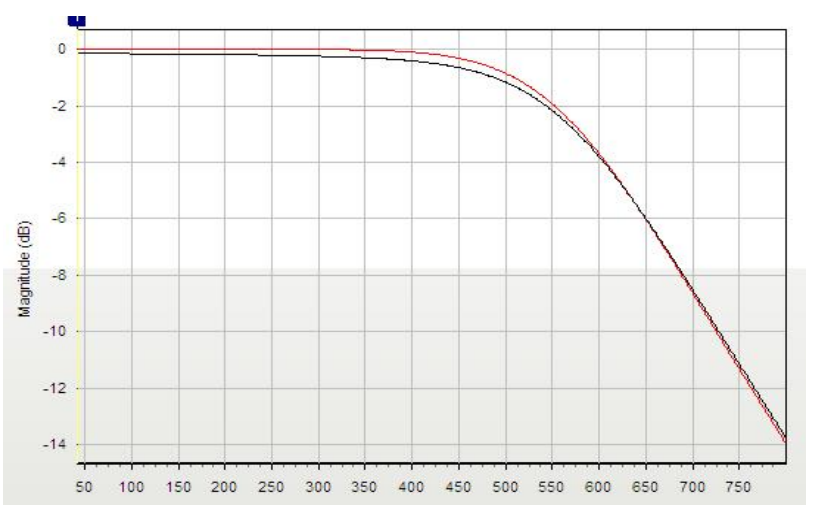

\section{Figure 5. Amplitude-frequency Response Curves of Analog Filter}

In general, the filter will produce a phase shift related with frequency. If the relationship of phase and frequency is linear, then the group delay is a constant. In subsequent processing, the real situation can be restored by offsetting the fixed delay time. However, if the phase characteristic is non-linear, it will make the serious phase distortion to non sinusoidal signal, which means that the filtered signal has deviation with the real situation ${ }^{[9-10]}$. It can be seen that the phase-characteristic in pass band is linear from Figure 6. 


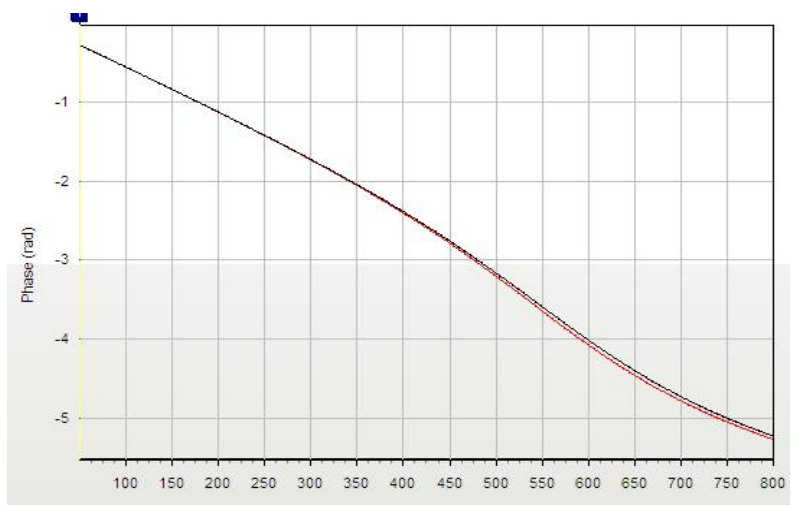

Figure 6. Phase-frequency Response Curve of Analog Filter

\section{The Data Processing Through Digital Filter}

Here, we design a FIR digital filter by FDAtool of Matlab to attenuate noise further. The pass band frequency of the digital filter is $400 \mathrm{~Hz}$, the stop band frequency is $430 \mathrm{~Hz}$ and the minimum stop band attenuation is $-30 \mathrm{~dB}$. The amplitude-frequency characteristic and the phase-frequency characteristic are shown in Figure 7. It can be seen that the filter has good steepness, stop band attenuation and strict linear phase in the pass band.

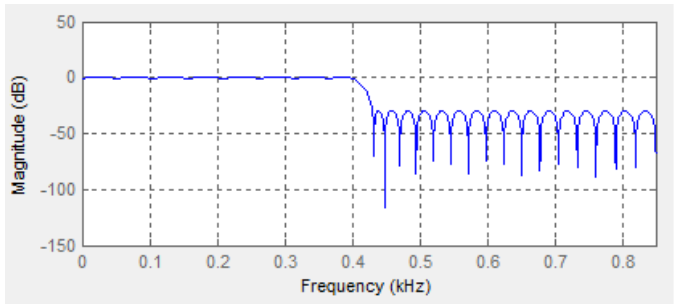

a) The amplitude frequency response

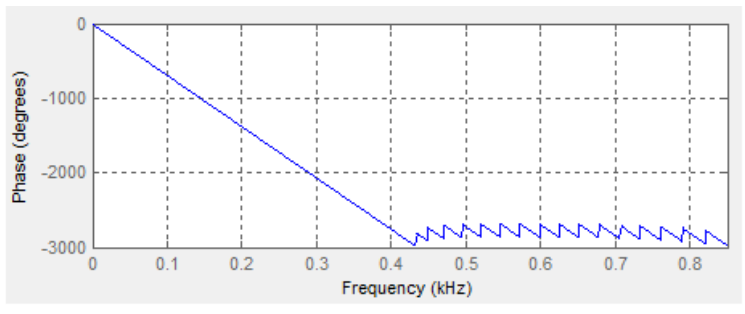

b) The phase frequency response

Figure 7. The Characteristics in Frequency Domain of Digital Filter

The data to be filtered is shown in Figure 8(a), the filtered data by anti-aliasing filter is shown in Figure 8(b). As can be seen that the wave in Figure 8(b) is much clearer than the wave in Figure 8 (a) and the clutter component in Figure 8(a) is eliminated because the stopband attenuation and the selective properties in the transition zone of the filter. That verifies that the filter can effectively suppress the high frequency signal of original contour and maintain the low frequency components well.

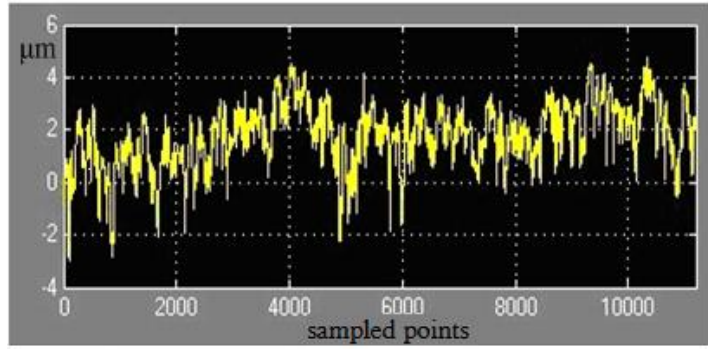

a) Waveform before filtering

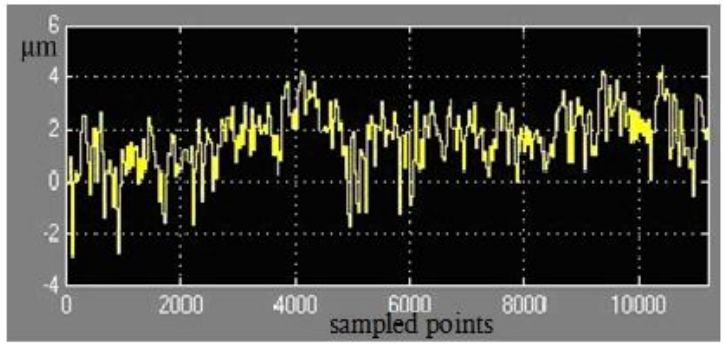

b) Waveform after filtering

Figure 8. Surface Profiles before Filtering and After Filtering 


\section{Conclusion}

This paper deals with the principle of anti-aliasing filtering and the design method of analog filter in detail. According to the measurement condition, the parameters of filter can be computed and the filtering circuit can be drawn by the tool software of Filter Wiz Pro. Through combination with the digital filter, the requirements for analog filter are lowered, it is easy to implement and the overall filtering effect is improved. The anti-aliasing filter can effectively suppress the noise over $400 \mathrm{~Hz}$ contained in the original profile, reduce the distortion caused by noise, and maintain the low frequency components very well, so the extracted surface roughness profile can more realistically reflect the actual surface situation.

\section{References}

[1] "ISO16610-2006 Geometrical product specifications (GPS)-Filtration-Part 1: Overview and basic concepts", International Organization for Standardization, Geneva, (2006).

[2] J. Raja, B. Muralikrishnan and S. Fu, "Recent advances in separation of roughness, waviness and form", Precision engineering, vol. 26, (2002), pp. 222-235.

[3] P. Dobrzanski and P. Pawlus, "Digital filtering of surface topography: Part I Part II", Precision engineering, vol. 34, (2010), pp. 647-658.

[4] C. Peiqing, "Digital signal processing course", Bei Jing: Third Edition, Tsinghua University Press, (2007). ( in Chinese)

[5] P. Yongsheng, W. Taiyong, F. Shengbo, W. Zhenyong and W. Shuangli, "Design of high quality anti-aliasing filter", Journal of southwest jiao tong university, vol. 38, no. 5, (2003), pp. 596-601. (in Chinese)

[6] S. Jun and Z. Mingzhong, "Method of analyzing and designing anti-aliasing filter", Modern electronics technique, no. 19, (2008), pp. 67-69. (in Chinese)

[7] T. G. Mathia, P. Pawlus and M. Wieczorowski, "Recent trends in surface metrology", Wear, no. 271, (2011), pp. 494-508.

[8] E. C. Tan and C. Y. Chia, "Numerical interpolations of analog and wave-digital elliptic filter parameters", Computers \& Electrical Engineering, no. 27, (2001), pp. 367-374.

[9] J. Shen, W. Shen, S. Castan and T. Zhang, "Sum-box technique for fast linear filtering", Signal processing, no. 82, (2002), pp. 1109-1126.

[10] S. Nalbalwar, S. D. Joshi and R. K. Patney, "On characterization of linear phase nonuniform filter banks", Signal Processing, no. 88, (2008), pp. 1980-1989. 